
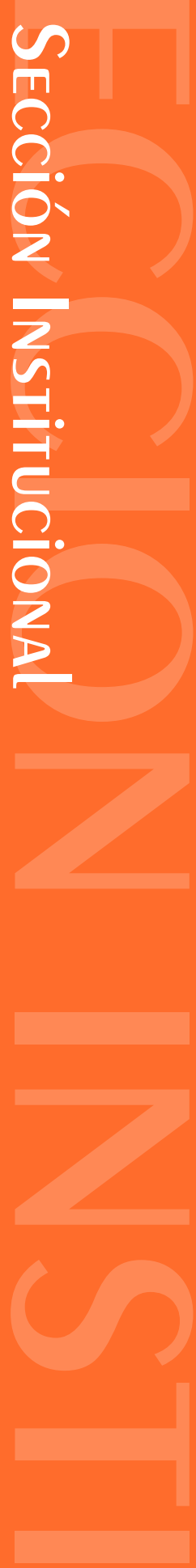

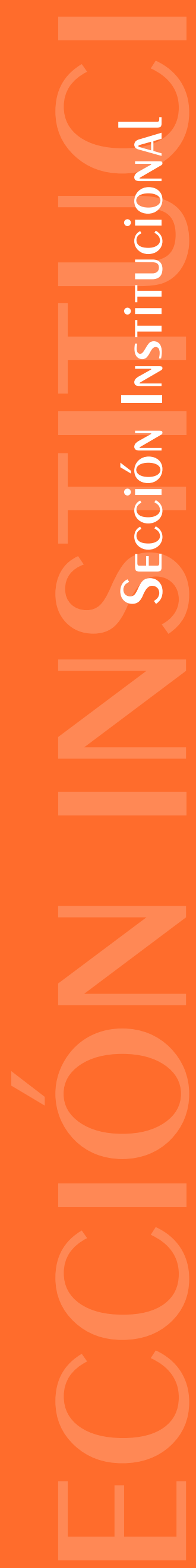


\title{
LA RECREACIÓN EN LA PRÁCTICA PEDAGÓGICA EDUCATIVA I Y II
}

\author{
RECREATION IN THE EDUCATIONAL TEACHING PRACTICE I AND II
}

\author{
Luz Jeanet Castañeda Quiroga \\ Viviana Sierra Delgadillo²
}

\section{Resumen}

El presente documento busca dar cuenta de las experiencias y los aprendizajes que se han construido en los procesos de práctica pedagógica I y II de la Licenciatura en Recreación y Turismo de la Universidad Pedagógica Nacional, entre 2010 y 2013, en algunos colegios públicos y privados de la ciudad de Bogotá; ello brinda también la oportunidad de hacer una mirada retrospectiva e indagar, cuestionar y reflexionar sobre los procesos y las dinámicas de estas prácticas en el proceso formativo del futuro docente. Se basa en la sistematización de los conocimientos previos y adquiridos tanto de los estudiantes como de los docentes que apoyan y orientan este proceso formativo, con el interés de indagar sobre los imaginarios que se tienen del futuro licenciado en recreación y su rol en la sociedad, y, más específicamente, en la escuela. Para la Universidad, como formadora de formadores, es importante generar espacios de transformación a partir de sus prácticas pedagógicas. Estas inciden en diseñar ambientes creativos, innovadores, propositivos, entre otros; pero también, donde los niños y los jóvenes no simplemente tengan acceso al derecho a la educación, sino que, más aun, sea este el escenario integral donde se brinden las condiciones para que puedan acceder de igual manera a otros derechos, tales como el derecho constitucional a la recreación. Por todo lo anterior, la práctica desde la Licenciatura en Recreación y Turismo debe permitir al docente en formación mirar si se ve a sí mismo como pedagogo, si realmente se involucra en los procesos didácticos que permitan la construcción del tejido social y si su saber recreativo le permite transcender e impactar en la escuela y en la sociedad. Por último, la reflexión se centra en analizar y autoevaluar los procesos metodológicos a partir de las prácticas pedagógicas I y II, y cómo estas posibilitan el reconocimiento de la recreación y el turismo como licenciatura en la escuela.

Palabras clave: práctica pedagógica, recreación, turismo, escuela, formación, lúdica y pedagogía

1 Magíster en Administración educativa. Profesora de planta, Secretaría de Educación del Distrito. Docente catedrática, Universidad Pedagógica Nacional, Licenciatura en Recreación y Turismo. Correo electrónico: jeanetcastaneda@yahoo.com

2 Licenciada en Recreación Aspirante maestrante en Educación. Docente de tiempo completo, Corporación universitaria CENDA, Licenciatura en Educación Física, Recreación y Deportes. Docente ocasional medio tiempo, Universidad Pedagógica Nacional, Licenciatura en Recreación y Turismo. Correo electrónico: vsierra20@gmail.com 


\section{Abstract}

This document proposes to explain the experiences and lessons that have been built in the processes of teaching practice I and II for the Degree in Recreation and Tourism of Universidad Pedagógica Nacional between 2010 and 2013, in some public and private schools in Bogota. It also provides an opportunity to make a retrospective look and inquire, question and reflect about the processes and dynamics of these practices in the training process of future teachers. It is based on the systematization of previous and acquired knowledge of both students and teachers who support and guide this formative process, with the interest of investigating imaginaries that have future graduates on recreation and their role in society, more specifically, at school. For the University, as a trainer of trainers, it is important to create transformation spaces based on their teaching practices. These have an influence on designing creative, innovative, proactive environments, among others; but also, where children and young people do not simply have access to right to education, but, moreover, it would be the integral stage where conditions are provided so they could access equally to other rights such as the constitutional right to recreation. For all these reasons, practice in the Degree in Recreation and Tourism should allow the trainees teacher to realize if he sees himself as a teacher, if he really gets involved in educational processes that allow construction of the social fabric and, also, if his recreational knowledge allows him to transcend and impact in school and society. Finally, reflection focuses on analyzing and self-assess the methodological processes from teaching practices I and II, and how these enable the recognition of recreation and tourism as degree in school.

Keywords: teaching practice, recreation, tourism, school, training, ludic and education

Fecha de recepción: 4 de diciembre de 2015

Fecha de aprobación: 4 de mayo de 2016

Para citar este artículo:

Castañeda, L. J. \& Sierra, V. (2016). La recreación en la práctica pedagógica educativa I y II. Lúdica Pedagógica, 24, 129-134.

\section{INTRODUCCIÓN}

Hablar de la práctica pedagógica en el programa de la Licenciatura en Recreación y Turismo que ofrece la Universidad Pedagógica Nacional permite hacer una mirada retrospectiva de los alcances y las limitaciones que han tenido los docentes en formación a lo largo del periodo 2010-2013 en algunos colegios públicos y privados de la ciudad de Bogotá; sobre todo, cuando este es el único programa académico que fragmenta la visión de la educación para el trabajo y se centra en la educación en los procesos pedagógicos que se pueden dar desde la recreación y el turismo.

En tal sentido, es importante mencionar que en el desarrollo curricular de la Licenciatura en Recreación y Turismo, la práctica no es vista como un espacio académico más, sino que, por el contrario, esta hace parte de todo el proceso formativo del docente en formación; es decir, desde los primeros espacios académicos, este futuro docente adquiere no solo las teorías correspon- dientes a su saber: además, tiene un acercamiento a esa realidad laboral, pero también social, en la cual se desempeñará en un futuro. Sin embargo, por la estructura administrativa de la universidad, se evidencia en el pénsum cuáles son esas prácticas que el estudiante va a realizar.

En este caso, la mirada se ha centrado en las dos primeras prácticas en las que el estudiante ya no solo observa, sino que, además de ello, analiza y propone una alternativa de trabajo para dar solución a una problemática que se dé en la comunidad. Para ello, el presente documento se dividirá en cuatro partes: la primera plantea un panorama contextual del proceso de práctica pedagógica I y II; la segunda presenta la institución educativa donde se lleva a cabo la práctica, y su relación con el docente en formación; la tercera hace referencia a la situación actual de la práctica educativa I y II en la Licenciatura en Recreación UPN y la cuarta aporta las reflexiones y las conclusiones del caso. 


\section{LA PRÁCTICA Y SU CONTEXTO}

Para dar inicio a esta contextualización, es importante comprender las dinámicas administrativas y sociales que se dan en la Universidad Pedagógica Nacional. Para ello, debe comprenderse lo que estipula el Acuerdo 035 de 2006, el cual establece que "la práctica pedagógica es el espacio de reflexión-acción e investigación en torno a la integración, innovación, indagación y recontextualización de los saberes de orden cognitivo, estético, pedagógico, didáctico y disciplinar en un contexto socio cultural especifico", pero, a su vez, cada programa académico está en libertad de proponer al Consejo Académico el reglamento de la práctica, así como los parámetros bajo los cuales se llevará a cabo el proceso formativo del futuro docente.

Desde la Licenciatura en Recreación y Turismo se aprobó, por parte del Consejo Académico, el reglamento de la práctica de la licenciatura que se encuentra establecido en el Acuerdo 018 de 2013, en el cual se disponen los objetivos, los principios y los escenarios de la práctica, al igual que sus modalidades. En el artículo 3 de dicho Acuerdo se establece que la práctica pedagógica educativa "se establece de forma directa a la licenciatura con instituciones o entidades de educación formal, de educación para el trabajo y desarrollo humano o informales"; así mismo, se menciona la cantidad de horas que se deben hacer de la práctica durante el semestre y la intensidad horaria que esta tiene.

Según el mismo Acuerdo, la práctica pedagógica educativa I tiene como objetivo "Aplicar y afianzar lenguajes ludo-recreativos con intención pedagógica en el contexto escolar"; en cuanto a la práctica pedagógica educativa II, se propone "Determinar problemáticas propias de la recreación en la escuela proponiendo formas de intervención". Con el ánimo de destacar lo vivido durante los ocho semestres transcurridos desde el Acuerdo, se describirán algunos de los apartes más relevantes que se han observado y evidenciado en las diferentes intervenciones realizadas en las instituciones educativas.

Cuando se da inicio a las prácticas educativas I y II en los colegios, los futuros licenciados en Recreación y Turismo enfrentan actitudes y aptitudes de desagrado por parte de los docentes y los coordinadores, al evidenciar que ellos no trabajan en su práctica la educación física, y, con mayor razón, cuando los niños o los jóvenes no son sacados al patio, como se acostumbra en esta área.

De igual manera, se evidencia que los docentes en formación en algunos lugares de praxis han sido tratados como si fueran estudiantes que prestan el servicio social, o bien fueron asignados a tareas que no les permitían enriquecer su quehacer pedagógico, lo cual generó malestar, insatisfacción e incertidumbre en los futuros docentes. Por lo tanto, se ve la necesidad de que los docentes formadores hagan mayor presencia en las instituciones educativas (IE), y que, a su vez, dentro del programa se posibiliten procesos reflexivos y críticos que lleven a identificar y posicionar poco a poco la labor formadora del licenciado en recreación y turismo; como primera medida, en la escuela, y como segunda, en otros escenarios donde este profesional se puede desempeñar.

Paralelamente a los diversos procesos del programa, surgieron cambios de orden administrativo, con los cuales se amplió la visión de la recreación y el turismo; esto se dio con la llegada de quien asumió la coordinación del programa académico para el periodo objeto de estudio, la licenciada Bibiana Rodríguez, quien vio la necesidad de fortalecer los conocimientos no solo de los futuros licenciados, sino también, los de los docentes que hacían el acompañamiento de la práctica. Para ello, se implementan diversas estrategias, tales como nombrar a un coordinador de práctica; quien asumió dicha labor fue el docente Pompilio Gutiérrez, y dentro de las tareas realizadas por él estuvo la consolidación del reglamento de práctica, al igual que realizar conversatorios, conferencias y talleres con docentes invitados nacionales e internacionales, como la doctora Guillermina Mesa, docente de la Universidad del Valle; el docente Ricardo Lema, de Uruguay; la doctora Esperanza Osorio, de la Fundación de Tiempo Libre (FUNLIBRE), y Guillermo Alfonso Forero Neira, en una alianza con el Servicio Nacional de Aprendizaje (SENA).

Todas las mencionadas estrategias contribuyeron a satisfacer las necesidades propias de la licenciatura, tales como posicionar a sus practicantes y buscar un reconocimiento de la recreación y el turismo dentro de la escuela. Estas estrategias han permitido ampliar el banco de instituciones; es decir, los lugares de práctica, donde los docentes en formación tienen el espacio real para poner en práctica los conocimientos adquiridos, 
así como para desarrollar habilidades y destrezas en clase, con el fin de cumplir con los objetivos propuestos.

Para los docentes en formación, no ha sido fácil romper los paradigmas establecidos por la sociedad acerca de la recreación y el turismo, demostrar, a partir de proyectos, nuevas estrategias o alternativas para atender las necesidades no solo educativas sino de los espacios de recreación propias de niños y jóvenes, mas no de la institución, ni de los docentes de aula; es decir, el centro de interés no es la lista de "necesidades de los administrativos o de los docentes", sino, por el contrario, las de los niños y los jóvenes con quienes se trabaja día a día. Desde esta perspectiva, se da paso a una aproximación a la metodología por proyectos como base fundamental para la creación de las propuestas de trabajo que los futuros licenciados presenten a las IE.

\section{LA IE Y SU RELACIÓN CON EL DOCENTE EN FORMACIÓN}

Encontrar el camino para las prácticas no ha sido sencillo; hacerlas de manera innovadora y creativa les ha permitido a los futuros docentes ir creando una brecha que los distinga de la práctica de educación física. La recreación y el turismo no son lo mismo que la educación física; de los docentes en formación se esperan propuestas innovadoras y creativas, que generen "procesos de formación alterativos, alternativos e incluyentes", elementos tomados del Documento maestro Licenciatura en Recreación (2007). Se espera también que dichas propuestas se diferencien de la educación física; sin embargo, "La recreación no excluye ni riñe con la educación física, sus caminos son distintos, tanto en sus saberes como en sus prácticas, lo cual no quiere decir, que estas tengan carácter exclusivo para uno u otro campo" (Carreño, Rodríguez \& Uribe, 2014, p. 139).

Al inicio de las prácticas, los docentes en formación se apoyan en diferentes manifestaciones y herramientas recreativas, tales como la ronda, el juego, el origami, la rumba recreativa, los cineforos, la danza, el arte circense, la creación de juguetes, la creación de instrumentos musicales, los títeres, los juegos autóctonos, las carreras de obstáculos, los juegos experienciales, los seguimientos de pistas, las dramatizaciones, los mimos y los rincones de interés, entre otros. Con todo ello, buscan explorar sus propias potencialidades y, por supuesto, las de la comunidad con la cual trabajan teniendo en mente que no se trata de un ejercicio de entretener o divertir al otro, sino de, más bien, comprender las dinámicas de una comunidad, sus problemas y sus fortalezas a partir de estos elementos, y sobre todo, comprender la manera como el licenciado en formación puede intervenir y construir en comunidad, como bien lo dice el Documento maestro de la Licenciatura (2007): "reconstruir el tejido social".

Algunas de las actividades mencionadas son propuestas por los niños y los jóvenes, y otras son implementadas por los docentes en formación. Cabe resaltar que ellos siempre se caracterizan por permitir que la comunidad dé paso libre a la creatividad y la imaginación desarrollando propuestas innovadoras y divertidas. Estas iniciativas han posibilitado que la recreación y el turismo sean pensados y reconocidos como un espacio de formación en la IE, y ello resalta su aporte en la construcción de ese ser integral que va para la sociedad.

Para que estos procesos se puedan dar, es importante reflexionar sobre las dificultades políticas, sociales y económicas que envuelven a las diferentes poblaciones infantiles y juveniles en el ámbito escolar. Esto no es fácil para los docentes ni para los practicantes, pero se deben generar espacios lúdicos-recreativos que les permitan a los niños y los jóvenes estimular su imaginación y su creatividad para brindar pequeños instantes de felicidad que rompan su cotidianidad; esto se basa en las experiencias observadas en las intervenciones de los practicantes, tales como Juan del Corral tiene talento, Pintucaritas, al igual que en la creación y la animación de títeres, las rumbas recreativas, los juegos tradicionales, las inauguraciones lúdico-recreativas intercursos y las manualidades.

"Se espera una escuela que evolucione desde la transformación de las personas, de sus modos de sentir, pensar y expresar su propia singularidad creativa, su potencialidad y recursividad, cuando de vivir se trata" (Acosta, Gutiérrez \& Rodríguez, 2010, p. 31). Varios proyectos de los licenciados en formación han tratado de satisfacer estas necesidades, lo cual es una de las funciones de los futuros docentes.

Se busca, a través de la práctica pedagógica educativa I y II de la Licenciatura en Recreación y Turismo, posibilitarles a los niños y los jóvenes una alternativa formativa, axiológica y lúdica que sirva como medio canalizador de su problemática cotidiana viabilizando una mejor 
calidad de vida para las poblaciones intervenidas, $y$, de igual manera, descubrir si el docente en formación tiene el perfil como formador a partir de la recreación y el turismo.

\section{SITUACIÓN ACTUAL DE LA PRÁCTICA EDUCATIVA I Y II DE LA LICENCIATURA EN RECREACIÓN Y TURISMO}

La experiencia que se ha ido madurando en las prácticas ha permitido a los docentes en formación visualizarse y visualizar la recreación y el turismo como un espacio formativo, interesante, motivador y generador de múltiples posibilidades en los procesos de formación.

Es tarea del docente acompañante guiar de manera intencionada las prácticas de recreación y turismo, y no permitir que se convierta en activismos improvisados, que no logran fortalecer la identidad de la Licenciatura en Recreación y Turismo ni darle un lugar en la escuela o en otros escenarios.

Es decir, la recreación que se reflexiona, discute, programa, diseña, gestiona, que hace parte de aquello que puede denominarse recreación intencionada, pedagógica, dirigida, orientada, entre otras posibilidades, pero que en general tiene que ver profundamente con la labor profesional de la recreación en cualquiera de sus niveles de la educación formal" (Carreño, Rodríguez \& Uribe, 2014, p. 137).

Debido a lo expuesto, fortalecer los procesos de diagnósticos, caracterizaciones, diarios de campo, planeaciones, evaluaciones y políticas educativas permite afianzar el quehacer de los futuros docentes. De la misma manera, la práctica pedagógica educativa I y II ha permitido ver el colegio como un espacio posibilitador de crecimiento docente, y no ponerse en el papel de criticar la institución educativa, sino de permitirse verla como una alternativa para "Desarrollar espacios de construcción académica alrededor de la pedagogía de la recreación y el turismo" (Documento maestro Licenciatura en Recreación y Turismo).

Para ello, a partir del segundo semestre de 2013, quien asumió la coordinación de la práctica docente, la licenciada Narda Robayo, propuso aumentar el número de docentes que conformaban el comité de práctica y la implementación de talleres que permitieran comple- mentar los conocimientos del futuro licenciado en recreación y turismo, buscando siempre proporcionar las herramientas necesarias para acercar lo más posible al licenciado en formación a la realidad laboral a la que se enfrenta el docente. Así, se implementaron talleres de danza, teatro, títeres, investigación, fotografía y música.

Lo anterior ha permitido generar otro tipo de dinámicas $\mathrm{y}$ discusiones en torno al proceso formativo del licenciado en recreación y turismo y su rol en el campo de la educación; dinámicas y discusiones que no se quedan solo en la academia, sino que, por el contrario, traspasan las barreras de lo teórico a lo práctico.

\section{CONCLUSIONES Y REFLEXIONES}

Uno de los aspectos más importantes del proceso de la práctica en el programa, y el cual es imperativo destacar, es que son los futuros docentes quienes agradecen la oportunidad que les brindan las instituciones educativas al permitir su ingreso a ellas, pues reconocen que este aspecto no es tan fácil de hacer como se podría pensar; las instituciones donde llevan a cabo las propuestas benefician a la comunidad en general, y en ellas se agradecen las orientaciones y las instrucciones que reciben por parte de los futuros docentes. Incluso, por parte de los rectores, los coordinadores, los docentes y los estudiantes se brindan apreciaciones interesantes, que permiten generar un espacio de crecimiento mutuo.

En la actualidad, las instituciones educativas acogen a los practicantes de la Licenciatura en Recreación y Turismo, los tienen en cuenta en la planeación anual para que intervengan en horarios académicos y desarrollen actividades transversales que beneficien a toda la población.

Se ha logrado fortalecer los conversatorios, las expectativas frente a la práctica y las propuestas que se presentan a las instituciones; se evidencian los logros y las dificultades en la praxis por medio de la socialización final, no solo en la institución donde se llevó a cabo la práctica, sino también, en la misma universidad. Para efectos de comprender a qué nos referimos con las socializaciones, las definimos como los medios que se han establecido para que los estudiantes muestren sus logros, sus dificultades y sus aprendizajes obtenidos durante el semestre; dichas socializaciones son presentadas por mediaciones recreativas (teatro, cuentería, 
ferias, etc.) o de manera formal, en cuyo caso los estudiantes muestran de diversas formas su sentir frente a lo trabajado en su práctica.

Con la implementación de los talleres (danza, teatro, títeres y fílmico-musical), los estudiantes se han declarado satisfechos, ya que estos les permiten fortalecer, aprender y afianzar otros conocimientos que les son útiles en las intervenciones con las diversas poblaciones que se atienden en las IE.

Los docentes que asesoran la práctica siempre han mantenido una actitud proactiva, propositiva y comprometida frente a los retos propuestos; así mismo, han procurado buscar otras alternativas que respondan no solo a las necesidades por parte de los estudiantes, sino también a las de la misma sociedad, frente al tema de la recreación, el ocio, el tiempo libre y el turismo.

En el transcurso de las prácticas, los docentes en formación inciden en algunas herramientas, pero se ha observado que son cada vez más creativos y ambiciosos en sus propuestas y crean retos interesantes; una muestra de ello son los proyectos que desarrollan.

A continuación, en la tabla 1 , se relacionan algunos de los proyectos implementados por los docentes en formación:

Tabla 1.

\begin{tabular}{|c|c|}
\hline Institución & Proyectos \\
\hline Colegio Juan del Corral IED & $\begin{array}{l}\text { Juan del Corral tiene Talento; } \\
\text { El cuerpo como instrumento } \\
\text { para la recreación; La recrea- } \\
\text { ción como proceso de formación } \\
\text { para el niño; Formando niños } \\
\text { felices a través de la creatividad, } \\
\text { la imaginación y el juego }\end{array}$ \\
\hline Colegio Magdalena Ortega IED & $\begin{array}{l}\text { Utilización de material reci- } \\
\text { clable: una propuesta para la } \\
\text { recreación }\end{array}$ \\
\hline Colegio Gustavo Restrepo IED & $\begin{array}{l}\text { Propuesta ludo-recreativa para } \\
\text { la estimulación de los cuadrantes } \\
\text { en el Colegio Gustavo Restrepo } \\
\text { IED }\end{array}$ \\
\hline $\begin{array}{l}\text { Liceo Nacional Agustín Nieto } \\
\text { Caballero IED }\end{array}$ & $\begin{array}{l}\text { Juegos tradicionales: una estra- } \\
\text { tegia de reflexión }\end{array}$ \\
\hline Gimnasio Santa Ana & $\begin{array}{l}\text { La recreación y la música: un } \\
\text { elemento de formación }\end{array}$ \\
\hline
\end{tabular}

\section{REFERENCIAS}

Acosta, J., Gutiérrez, P. \& Rodríguez, A. B. (2010). La recreación en la escuela: un campo de exploración y gestión de la imaginación. Lúdica Pedagógica, 2 (15), $27-33$.

Carreño, J. M., Rodríguez, A. B. \& Uribe, J. J. (2014). Recreación, Ocio y Formación. Armenia: Kinesis.

Documento maestro Licenciatura en Recreación (2007). Facultad de Educación Física. Resolución 1659 del 19 abril del 2006. 